\title{
Actuaciones educativas de éxito desde la Educación Física Educative actions for success in Physical Education
}

\author{
Adriana Aubert", Mariate Bizkarra**, Jordi Calvo* \\ *Universitat de Barcelona. **Universitat del País Vasco.
}

\begin{abstract}
Resumen: El artículo que se presenta a continuación sirve como introducción al monográfico y propone un marco teórico general sobre el cual el conjunto de artículos adquieren su significado. En él se define y justifica el concepto de actuaciones educativas de éxito que se contextualiza en el área de educación física en el marco de las Comunidades de Aprendizaje. Todas las propuestas que se presentan son el resultado de investigaciones llevadas a cabo en centros educativos. Algunas han sido identificadas por el proyecto INCLUD-ED (2009), el único de Ciencias Sociales seleccionado por la Comisión Europea en su lista de las 10 mejores investigaciones científicas (European Commission, 2011a), por su contribución a la superación del fracaso escolar y mejora de la convivencia en los centros. Se destacan entre ellas la resolución dialógica de los conflictos, la organización de la clase en grupos interactivos, o la promoción de la salud. La mayoría de las contribuciones que constituyen el presente monográfico están relacionadas con el proyecto de investigación: Juega, Dialoga y Resuelve: La superación de conflictos en Educación Física mediante el modelo comunitario. Diseño de un programa específico para Comunidades de Aprendizaje perteneciente al Plan Nacional I+D+i 2007-2009). El resto, están, bien vinculadas a la prevención y resolución de los conflictos a través del área de educación física, bien al proyecto de Comunidades de Aprendizaje. Este artículo introductorio ofrece, además, una exhaustiva síntesis de cada una de estas actuaciones educativas de éxito que configuran el conjunto del monográfico. Palabras clave: educación física, actuaciones educativas de éxito, comunidades de aprendizaje.
\end{abstract}

\begin{abstract}
The paper presented below is showed as introductory at the monograph and proposes a theoretical framework on which the set of articles of the monograph take their meaning. Thus, the concept of educational actions of success is justified and explained and contextualized in the Physical Education at the Learning Communities context. All proposals presented are the result of research developed in schools. Some of them have been identified by the project INCLUD-ED (2009), which is the only Social Sciences project selected by the European Commission in the list of the 10 best scientific research projects (European Commission, 2011a), for its contribution in overcoming school failure and improving behavior in schools. Among the educational actions of success the dialogical conflict prevention, the class organization in interactive groups and health promotion are emphasized. Most of the contributions that form the present monograph are related to the research project: Juega, Dialoga y Resuelve: La superación de conflictos en Educación Física mediante el modelo comunitario. Diseño de un programa específico para Comunidades de Aprendizaje (Plan Nacional I+D+i 2007-2009: SEJ2007-61757/EDUC). The rest of the papers are linked to prevention and resolution of conflicts through Physical Education in Learning Communities. This introductory article also provides an exhaustive summary of each educational action of success that forms the whole monograph.
\end{abstract}

Keywords: Physical Education, Educative actions for success, Learning Communities.

\section{Introducción}

Entendemos por actuaciones educativas de éxito aquellas que se orientan a la transformación social y que contribuyen a la superación del fracaso escolar, como las que se están realizando en aquellos centros que se han transformado en Comunidades de Aprendizaje. Entre estas actuaciones, se destacan aquellas vinculadas con la resolución dialógica de los conflictos, la organización de grupos interactivos para el desarrollo del aprendizaje dialógico en educación física, o estrategias para la promoción de la salud en la escuela. Tales actuaciones han sido identificadas y analizadas en el proyecto INCLUD-ED (2009) considerado como una de las mejores investigaciones científicas en Ciencias Sociales por la Comisión Europea (2011a).

Entre estas actuaciones, en este monográfico, se presta mayor atención a las relacionadas con la prevención dialógica de los conflictos vinculados al proyecto de investigación: Juega, Dialoga y Resuelve: La superación de conflictos en Educación Física mediante el modelo comunitario. Diseño de un programa específico para Comunidades de Aprendizaje. (Plan Nacional I+D+i 2007-2009: SEJ2007-61757/EDUC). Acompañan este monográfico también un estudio sobre la consecución de la competencia social y ciudadana, y un estudio sobre la promoción de la salud. Todo ello con la intención de responder a las demandas específicas de los centros que son Comunidades de Aprendizaje sobre el tratamiento de la educación física, en coherencia con el aprendizaje dialógico, y los requerimientos de la escuela en la sociedad de la información.

Fecha recepción: 05-12-13- Fecha envío revisores: 05-12-13- Fecha de aceptación: 10-12-13 Marta Capllonch Bujosa

Campus Mundet, Edifici Llevant, 3a planta, despatx 317,

Passeig del Vall d'Hebron 171, 08035 Barcelona,

mcapllonch@ub.edu
La educación física en la sociedad de la información

En el apartado de introducción, hemos puesto de manifiesto que este monográfico presta especial atención a las actuaciones educativas deéxito relacionadas con la prevención dialógica de los conflictos. Y es éste, de hecho, uno de los argumentos que justifica la vinculación de la investigación con el área de educación física: tal y como queda recogido en los artículos posteriores, se puede considerar que esta área curricular representa un espacio privilegiado para el tratamiento del conflicto escolar (Capllonch, 2008-2011). Pero, más allá de esta cuestión, hemos considerado conveniente incluir una breve reflexión sobre la relevancia de la educación física en la escuela actual; una escuela inmersa en la denominada sociedad de la información. Cabe destacar que el papel de la educación física en la escuela ha sido cuestionado en diversos momentos; $y$ precisamente por eso podemos encontrar aportaciones de expertos del área que reflexionan sobre esta cuestión y exponen sus propuestas al respecto (Blanquerna-FIEP Catalunya, 2008).

Así pues, a partir de la visión de los autores que recoge el documento (Blanquerna-FIEP Catalunya, 2008), podemos ofrecer un conjunto de argumentos que muestran la necesidad, la conveniencia, el porquéde la educación física como materia curricular en el sistema educativo. A saber: a) la educación física es un elemento socializador que favorece la cohesión social; b) promueve los hábitos saludables y mejora la calidad de vida; c) sirve de guía y acompañamiento para un desarrollo motor armónico; d) actúa como elemento dinamizador de los centros educativos y enseña una manera de vivir el tiempo libre; e) interviene en la construcción de la inteligencia humana a través de la motricidad; f) favorece la comunicación corporal.

Desde esta perspectiva, que considera que la educación física tiene un papel importante en el proceso educativo de los niños y las niñas, resulta también pertinente querer avanzar hacia actuaciones educativas de éxito desarrolladas en el contexto de esta área curricular. Y este planteamiento nos lleva, pues, a considerar la educación física dentro del marco que definen las comunidades de aprendizaje; que, como veremos 
a continuación, constituyen un elemento de referencia en el ámbito de las actuaciones de éxito.

\section{Las Comunidades de Aprendizaje}

Las Comunidades de Aprendizaje (CdA) están desarrollando las Actuaciones Educativas de Éxito (AEE) avaladas por la Comunidad Científica Internacional (CCI) con el objetivo de lograr, por un lado, el aumento del aprendizaje de todo el alumnado y, por otro, la mejora de la convivencia. Las AEE se caracterizan por haber obtenido las mayores mejoras allá donde se hayan desarrollado con los mismos recursos disponibles en cada entorno, y se concretan en los grupos interactivos como forma inclusora de organizar el alumnado; las tertulias dialógicas desde las que se fomenta el aprendizaje de la lectura y el acceso al conocimiento científico acumulado por la humanidad a lo largo del tiempo; la formación de familiares que influye de forma directa en el rendimiento escolar del alumnado; la participación educativa de la comunidad en las actividades de aprendizaje del alumnado tanto en el horario escolar como fuera; la formación dialógica del profesorado que contribuye enormemente al paso de las ocurrencias a las evidencias en educación y; el modelo dialógico de prevención y resolución de conflictos.

Este proyecto de transformación social y cultural de un centro educativo y de su entorno parte de las teorías científicas más relevantes (la Teoría de la Acción Dialógica de Freire, 1970; la Teoría de la Acción Comunicativa de Habermas, 1987; el interaccionismo simbólico de Mead, 1973; y la teoría histórico-cultural de Vygotsky, 1996; entre otras y otros) y se basa en el aprendizaje dialógico desarrollado en y para la sociedad de la información (Flecha, 1997). En el marco de la concepción comunicativa, el aprendizaje dialógico pone el foco en la dimensión intersubjetiva del aprendizaje, entendiendo la construcción del conocimiento como un proceso que se da en relación y comunicación con las demás personas con las que interactuamos. A través de sus siete principios (el diálogo igualitario, la inteligencia cultural, la transformación, la dimensión instrumental, la creación de sentido, la solidaridad y la igualdad de diferencias) se llevan a cabo las AEE, abriendo todos los espacios del centro a la participación de la comunidad, incluida el aula.

Cada vez con mayor rapidez centros educativos de diferentes entornos socioeconómicos y etapas educativas, tanto de España como de diversos países de Latinoamérica, se van sumando a las más de 150 CdA que existen actualmente. A su vez, diversos gobiernos autonómicos ofrecen su apoyo y cobertura, y se han creado redes muy consolidadas en diferentes zonas.

El proyecto de investigación INCLUD-ED (2009) el único de Ciencias Sociales seleccionado por la Comisión Europea en su lista de las 10mejores investigaciones científicas (European Commission, 2011a), ha identificado y analizado las AEE que desarrollan las CdA. El impacto de la mejora de resultados que esta investigación evidencia se ha traducido en diversas resoluciones de la Comisión Europea y del Consejo de Europa desde las que se recomienda la transformación de los centros educativos en CdA (European Commission, 2011b; Council of the European Union, 2011; European Parliament, 2009), así como en la publicación de sus resultados en revistas científicas tales como la de Harvard o Cambridge.

\section{El modelo dialógico de prevención y resolución de conflictos}

En las últimas décadas ha cambiado el modo en cómo nos relacionamos unas personas con otras. Cada vez más el diálogo va penetrando en todos los espacios de la sociedad sustituyendo la imposición de la violencia física o simbólica. Las antiguas normas y patrones que guiaban nuestras vidas en la sociedad industrial han ido perdiendo legitimidad y las personas recurrimos de forma creciente al diálogo para resolver conflictos y para tomar decisiones.

En el seno de la escuela, a menudo se pide a las familias y a la comunidad educativa que acepten unas normas impuestas. La paz tampoco se logra desde una perspectiva postmoderna en la que se impone un relativismo que no considera que haya valores mejores ni peores y que define todas las relaciones como si fueran de poder. Tanto este relativismo como la caduca modernidad tradicional han dado paso a procesos basados en pretensiones de validez (Habermas 1998) en los que se consensuan conjuntamente las transformaciones a hacer en el centro, que lleven a la defensa de unas normas compartidas y decididas por todos y todas. El giro dialógico que se ha dado en la sociedad actual explica por qué la paz no se puede imponer a través de pretensiones de poder, sino que se debe consensuar y defender activamente por todos los agentes educativos, poniendo en común los deseos, intereses e inquietudes, y asentando los valores en un diálogo entre una creciente pluralidad de voces.

Dado este contexto, se hace necesario superar el modelo disciplinar de resolución de conflictos basado en una autoridad que decide e impone unas normas, así como las medidas sancionadoras para hacerlas cumplir. También plantea la necesidad de ir más allá del modelo «experto/a» desde el que se favorece la comunicación entre las personas involucradas en un conflicto, buscando vías de solución que se basen en la negociación y no en la intervención punitiva. El modelo dialógico de prevención y resolución de conflictos, en el marco de la concepción comunicativa de la sociedad, involucra a toda la comunidad para encontrar las causas del conflicto y las formas de superarlo. El que el centro sea un lugar seguro es un deseo compartido por toda la comunidad, un deseo común que asienta las posibilidades para llegar a consensos entre personas de muy diversas identidades, orígenes, intereses y condiciones.

Por ello, los centros que desarrollan este modelo de prevención de conflictos empiezan consensuando una norma que deberá cumplir una serie de condiciones: 1) que pueda ser claramente acordada por todas las personas, de todas las mentalidades y edades; 2) Que tenga relación directa con un tema clave para las vidas de las niñas y niños; 3) Que haya apoyo «verbal» claro del conjunto de la sociedad; 4) Que se incumpla reiteradamente; 5) Que se vea posible eliminarlo; 6) Que con su superación, la comunidad dé un ejemplo a la sociedad, familiares, profesorado, niñas y niños. Una vez acordada esta norma, se siguen una serie de pasos para lograr que sea toda la comunidad quien vele por su cumplimiento y haga un seguimiento de forma permanente.

De forma complementaria a la mejora de la convivencia que supone la organización del aula en grupos interactivos, por ejemplo, o los debates que se generan en torno a los libros de la literatura clásica universal que lee el alumnado en las tertulias literarias dialógicas, o la participación educativa de las familias que entran en las aulas para ayudar al profesorado, entre otras AEE, el modelo dialógico de prevención de conflictos está dando una mejora significativa de la convivencia.

\section{Objetivos}

El objetivo del presente monográfico se centra en presentar algunas actuaciones educativas de éxito que se están llevando a cabo en elárea de educación física, avaladas por la comunidad científica internacional. Se presta especial atención a aquellas vinculadas con la prevención y resolución dialógica de los conflictos, desarrolladas en centros que son Comunidades de Aprendizaje.

Actuaciones educativas de éxito desde la educación física. Presentación de los artículos del monográfico

Este monográfico organizado en torno a la superación de conflictos en educación física como eje principal, quiere representar una reflexión sobre cómo puede la educación física, como área curricular, contribuir a la superación del fracaso escolar en la escuela, la cohesión social y la mejora de la convivencia en los centros. Comienza con este primer artículo introductorio en el que se plantea la definición de actuaciones educativas de éxito, y el valor que están adquiriendo los centros que son comunidades de aprendizaje para avanzar en el conocimiento y la mejora del aprendizaje.

El segundo artículo titulado «Prevención y resolución de conflictos en educación física: estado de la cuestión» ofreceuna reflexión bibliográfica sobre los antecedentes de la prevención y resolución de conflictos en esta materia, y un análisis del estado de la cuestión. En dicho artículo se revisan las investigaciones referidas en las bases de datos más impor- 
tantes en el área de Ciencias Sociales, tales como, ISOC, TESEO, ISI y ERIC. Se quiere con ello, conocer los modos de aproximarseal conflicto, y se analiza la necesidad de abordar las temáticas de prevención y resolución de la conflictividad, en el área de educación física. Se establecen diferentes criterios de búsqueda a través de palabras clave como: resolución de conflictos, educación física y resolución de conflictos, deporte y valores, educación física y valores, educación física e integración cultural, comunidades de aprendizaje y resolución de conflictos, y responsabilidad personal y social en educación física. Así, se recoge que una de las referencias de mayor calado es la del modelo del programa de responsabilidad personal y social de Hellison (1995, 1999, 2003). Por otra parte se destacan las Comunidades de Aprendizaje, que por su manera colegiada de trabajar el conflicto se muestran como un escenario privilegiado para realizar acciones coordinadas con las diferentes personas de la comunidad (Flecha \& García Yeste, 2007; Vargas \& Flecha, 2000).

El tercer artículo que lleva por título «Tratamiento del conflicto en las Comunidades de Aprendizaje a través de la educación física», muestra como los conflictos que se producen en la escuela, y cómo superarlos, es uno de los temas que más preocupa en las Comunidades de Aprendizaje. Para su superación y prevención, algunas de estas escuelas están desarrollando un modelo comunitario de resolución de conflictos que está teniendo un impacto positivo en los problemas que surgen en las clases de educación física, al fomentar la participación de las diferentes personas de la comunidad mediante procesos de diálogo y decisión sobre las normas de convivencia(Vizcarra, Macazaga \& Rekalde, 2013). Los conflictos aparecen como una oportunidad para la mejora del grupo (Molina, 2005; Ortí, 2003), ya que las conductas agresivas se dan con mayor frecuencia en los espacios que hay menor vigilia institucional como pueden ser el patio, o incluso el aula de educación física (Macazaga, Rekalde \& Vizcarra, 2013). El modelo comunitario para el tratamiento de los conflictos aparece como una solución ideal ante situaciones en las que el mayor problema es la jerarquía (Aubert, Duque, Fisa \& Valls, 2004). En los resultados se aprecia que el alumnado no ve la Educación física como un área especialmente conflictiva. Los principales problemas de convivencia en el área se dan como consecuencia de las trampas en los juegos, la exclusión por el nivel de habilidad, la excesiva competitividad o las faltas de respeto a las normas de juego. Se intuye y se comparte entre los diferentes miembros de la comunidad (alumnado, profesorado, familiares y voluntariado), la importancia del diálogo y el consenso para llegar a acuerdos, y la necesidad de superar la toma unilateral de decisiones por parte del profesorado, no solo en las clases de educación física, sino en toda la escuela.

En el cuarto artículo: «Prevención y resolución del conflicto en educación física desde la perspectiva del alumnado», se analiza cómo este enfrenta la resolución de los problemas que surgen en el área de educación física. En primer lugar se define el conflicto como una experiencia negativa porque a menudo se trata de imponer una visión o una opinión y se olvida la posibilidad de dialogar y de intentar ver el punto de vista del «otro», sin embargo la exposición de los modelos de intervención que en este momento conviven en los centros educativos para la prevención y resolución de los conflictos apuntan hacia la posibilidad de llegar a consensos mediante el diálogo que están mejorando la convivencia en el aula y en todos los espacios del centro (Aubert, et al., 2004). Así desde esta perspectiva el artículo muestra como el alumnado no percibe el área de EF como especialmente conflictiva, sin embargo muestra su desacuerdo ante comportamientos inadecuados que observan en sus centros educativos. Entre las razones detonantes del conflicto que son más destacables en las clases de EF aparecen el género, la exclusión por el nivel de habilidad y la excesiva búsqueda de la victoria. Destacan que el conflicto adopta diferentes formas entre ellas: discusiones, agresiones verbales y/o físicas, injusticias, difamaciones e indisciplina como las opciones elegidas mayoritariamente. Sus reacciones ante el conflicto suelen ser de exclusión o de crispación, aunque empieza a entreverse una voluntad por parte de todos los miembros de la comunidad, de llegar a consensos entre todos y todas. En las clases de EF se propone llevar a cabo actividades cooperativas en las que todos trabajan para alcanzar un objetivo común, el reconocimiento de las diferencias, y la inclusión de los grupos interactivos (Capllonch, 2008-2011).

En el artículo quinto, que lleva por título: «Prevención y resolución del conflicto en educación física desde la perspectiva de los miembros adultos de la comunidad educativa en las comunidades de aprendizaje», se analizan las opiniones, creencias y valoraciones que los miembros adultos (profesorado, familiares y voluntariado) de las Comunidades de Aprendizaje realizan sobre las causas de los conflictos que se producen en las clases de educación física, y la forma de abordarlos (Capllonch, 2008-2011). Conocer esta información, ayudará a orientar aquellas acciones que favorezcan el tratamiento adecuado del conflicto. En la fundamentación teórica se recogen las disertaciones de Vygotsky (1978) sobre la importancia de que las niñas y niños cuenten en su entorno con personas adultas de referencia que les ayuden a mejorar en su aprendizaje (Tellado y Sava, 2010). Así se ve que la educación física es un área curricular, que representa un espacio privilegiado para el tratamiento y prevención del conflicto escolar(Vizcarra, Macazaga, Perera, Maiztegui, Arostegui \& Gasituaga, 2007). Según la apercepción de profesorado, familiares y voluntariado, las casusas del conflicto en educación física están provocadas por la desigualdad de oportunidades para conseguir el éxito, la reproducción de actitudes que se ven en los medios de comunicación, la casi exclusiva manera de resolver los conflictos de manera violenta o aplicando principios de autoridad, y las dificultades en la comunicación. Como posibles formas de abordar estos problemas se plantean estrategias como la de consensuar normas de funcionamiento entre todos y todas (Aubert, et al., 2004), orientar el área de educación física hacia adquisición de valores (Velázquez Callado, 2003, 2004) o gestionar adecuadamente la competición para que todos y todas tengan las mismas oportunidades (Velázquez Buendía, 2001).

En el sexto artículo titulado: «Aprendizaje dialógico y grupos interactivos en educación física», se explican los principios del aprendizaje dialógico y de los grupos interactivos en educación física, para lo que se han tenido en cuenta las opiniones del alumnado, profesorado, voluntariado y familias de seis comunidades de aprendizaje, tres del País Vasco y tres de Cataluña. El aprendizaje dialógico se sustenta sobre la idea de que lo que se aprende depende cada vez menos de lo que sucede en el aula, y toma importancia la información recogida de la red, del grupo de iguales, de sus familias, etc. La noción de aprendizaje dialógico nace del análisis de la sociedad de la información y de los proyectos de las Comunidades de Aprendizaje (Aubert, Flecha, Flecha, García \& Racionero, 2008; Flecha, 1997; Flecha \& García, 2007) y se basa en principios tales como la intersubjetividad de los aprendizajes, el valor de los argumentos por su validez, y no por la posición que ocupa que quien los postula... el diálogo es por tanto, igualitario, analizando las situaciones de desigualdad con el fin de transformarlas, mediante tareas solidarias enfocadas hacia el bienestar del alumnado. En este sentido, adquieren especial relevancia los grupos interactivos, que utilizan formas no tradicionales de agrupación del alumnado. Para ello se organizan pequeños grupos heterogéneos en todas las dimensiones posibles (género, nivel de aprendizaje, origen cultural, afinidad...) que son tutorizados por una persona adulta voluntaria (Elboj \& Niemela, 2010, Galobardes, 2010, Kirikiades \& Valls). La recogida de información se realizó desde una metodología comunicativa crítica orientada a la construcción de conocimiento desde la intersubjetividad y la reflexión conjunta con los sujetos de la investigación directamente implicados (Gómez, Latorre, Sánchez \& Flecha, 2006).

El séptimo artículo que lleva por título «La educación física en la consecución de la competencia social y ciudadana», recoge el resultado de una investigación-acción que estudia la necesidad de cambiar el clima de convivencia de un centro de educación primaria. Se deseaba conocer en qué medida podía contribuir el trabajo de la competencia social y ciudadana en la prevención y resolución pacífica de los conflictos desde el área de Educación Física. Una vez revisadas las investigaciones de la última década en actividad física y resolución de conflictos (Cecchini, Montero \& Peña, 2003; Escartí, Pascual \& Gutiérrez, 2005; Hellison, 1995; Jiménez \& Durán, 2004; Monjas, 2008; Vizcarra, 2004), se recogieron los resultados a través de cuestionarios y mediante utilización de 
la observación participante, tratando así, datos cualitativos y cuantitativos. Se pudo constatar que tras la intervención se minimizaron las resoluciones que conllevaban violencia y aumentaba el diálogo autónomo. Así, el alumnado destacó que son las trampas, la competitividad y la excesiva agresividad las que inciden demanera negativa en el desarrollo las actividades en las clases de educación física. Serecogió asimismo que, ante el conflicto, las actitudes más habituales eran ignorar el hecho conflictivo, comentarlo con las personas adultas o intentar solucionar el problema a través del diálogo.

En el octavo y último artículo, «Educación física y promoción de la salud: estrategias de intervención en la escuela» se muestra que la prevención de la salud en educación física puede ser una oportunidad para fomentar un desarrollo social en ambientes menos favorecidos. De tal manera, que se realiza la revisión de la literatura científica internacional sobre programas de salud en contextos educativos, que estén centrados especialmente en educación primaria, y vinculados a la educación física, mediante una búsqueda en bases de datos de publicaciones de gran impacto internacional. El estudio está amparado en el proyecto de investigación INCLUD-ED. Strategies for inclusion and social cohesion fromeducation in Europe, del Sexto Programa Marco de Investigación de la Unión Europea, y enaltece la Educación Física como un área con enorme potencial en el desarrollo de hábitos para la promoción de la salud, y el desarrollo del bienestar del alumnado. Incide en el papel que juega la educación física en la promoción de la salud en los grupos de población más vulnerables. Y esto es posible, si esta área de conocimiento es entendida como una actividad que traspasa los muros de las aulas y llega a toda la comunidad, encontrando en ella una oportunidad para acceder al aprendizaje por parte de toda la Comunidad (DíezPalomar \& Flecha, 2010). En este estudio se observa que la participación regular en actividades físico-deportivas conlleva una mejor calidad de vida, ya que se palian los riesgos de padecer enfermedades psicológicas y cardiovasculares (Koh, Piotrowski, Kumanyika \& Fielding, 2011). Otra de las preocupaciones que pone al descubierto este estudio es la inactividad física de niños y jóvenes en España, ya que ésta se encuentra por debajo de la media europea, albergando un elevado índice de sedentarismo, ya que según se ha estimado, en el año 2015, uno de cada cinco niños tendrá sobrepeso (Janssen, Toussaint, Van Willem \& Verhagen, 2011). En los resultados se presentan las investigaciones agrupadas en diferentes líneas de investigación, y así, se observa la presencia de estudios que se sirven de las nuevas tecnologías para la promoción de la salud, constituyendo ésta una línea de investigación de reciente aparición, con una amplia expectativa de desarrollo en un futuro próximo, tal y como destacan algunos autores (Tercyak, Abraham, Graham, Wilson \& Walker, 2009); la presencia de estudios realizados desde una perspectiva preventiva (Telford, et al., 2009); la presencia de estudios que incluyen la perspectiva de la evaluación y eficacia, presentando programas de prevención de la obesidad escolar, (Kain, Leyton, Cerda, Vio \& Uauy, 2009; de Meij, et al., 2010): los estudios que incluyen una perspectiva de comunidad educativa como agente en el desarrollo de los programas curriculares de educación física como factor que desarrolla la salud (Thorburn, Carse, Jess \& Atencio, 2011). Estudios que incluyen la perspectiva del contexto (de Bourdeaudhuij, et al. 2011) que señalan que la combinación de componentes educativos y ambientales inciden en la promoción de la actividad física; y finalmente, estudios centrados en comunidades vulnerables (Pappa, Kontodimopoulos, Papadopoulos \& Niakas, 2009).

En conclusión, el presente monográfico es una muestra de cómo la educación física puede contribuir, desde diferentes ámbitos como pueden ser el aprendizaje dialógico, los grupos interactivos, la prevención dialógica de los conflictos, o la promoción de la salud, a desarrollar actuaciones que garanticen el éxito escolar, y con ello contribuir desde su especificidad a la superación del fracaso escolar.

\section{Referencias}

Aubert, A., Duque, E., Fisas, M., \& Valls, R. (2004). Dialogar y transformar. Pedagogía crítica del siglo XXI. Barcelona: Graó.
Aubert, A., Flecha, A., García Yeste, C., Flecha, R., \& Racionero, S. (2008). Aprendizaje dialógico en la Sociedad de la Información. Barcelona: Hipatia.

Blanquerna-FIEP Catalunya. (2008). Els perquès de l’Educació Física avui. EnIV Jornada Esport Blanquerna-V Jornada FIEP Catalunya. Barcelona: Universitat Ramon Llull.

Capllonch, M. (2008-2011). Juega Dialoga y Resuelve. La superación de conflictos en educación física mediante el modelo comunitario. Diseño de un programa específico para comunidades de aprendizaje, con referencia, con referencia SEJ2007-61757/EDUC. Plan Nacional I+D+I.

Cecchini, J. A., Montero, J., \& Peña, V. (2003). Repercusiones del Programa de Intervención para desarrollar la Responsabilidad Personal y Social sobre los comportamientos de fair-play y el autocontrol. Psicothema(15), 631-637.

Council of the Eurpean Union. (2011). Council Recommendation on policies to reduce early school leaving. (Text with EEArelevance). (2011/C 191/01). (http://ec.europa.eu/education/school-education/ doc/earlyrec_en.pdf).

De Bourdeaudhuij, I., Van Cauwenberghe, E., Spittaels, H., Oppert, J. M., Rostami, C.,. . . \& Maes, L. (2011). School-based interventions promoting both physical activity and healthy eating in Europe: a systematic review within the HOPE project. Obesity Reviews, 12(3), 205-216. doi: 10.1111/j.1467-789X.2009.00711.X

De Meij, J.S.B., Chinapaw, M.J.M., Kremers, S.P.J., Van der Wal, M.F., Jurg, M.E., \& Van Mechelen, W. (2010). Promoting physical activity in children: the stepwise development of the primary school-based JUMP-in intervention applying the RE-AIM evaluation framework. British Journal of Sports Medicine, 44(12), 879-887. doi: 10.1136/bjsm.2008.053827

Díez-Palomar, J., \& Flecha, R. (2010). Comunidades de Aprendizaje: un proyecto de transformación social y educativa. Revista Interuniversitaria de Formación del Profesorado, 67(24,1), 1930.

Elboj, C., \& Niemela, R. (2010). Sub-communities of mutual learners in the classroom: the case of Interactive Groups. Revista de Psicodidáctica, 15(2), 177-189.

Escartí, A., Pascual, C., \& Gutiérrez, M. (2005). Responsabilidad personal y social a través de la educación física yel deporte. Barcelona: Graó.

European Commission (2011a). Added value of Research, Innovation and Science portfolio (MEMO/11/520 19/07/2011). Recuperado de http://europa.eu/rapid/press-release_MEMO-11-520_en.htm.

European Commission. (2011b). Communication from the Comission to the European Parliament, the Council, the European Economic and Social Committee and the Comitte of the regions. Tackling early school leaving: A key contribution to the Europe 2020 Agen$d a$. Recuperado dehttp://ec.europa.eu/education/school-education/ doc/earlycom_en.pdf.

European Parliament. (2009). Educating the children of migrants. European Parliament resolution of 2 April 2009 on educating the children of migrants. (2008/2328(INI). Recuperado de http://eur$\mathrm{l}$ e $\mathrm{x}$. e u r o p a . e u/L e x U r i S e r v / LexUriServ.do?uri=OJ:C:2010:135:0002:0007:EN:PDF.

Flecha, R. (1997). Compartiendo palabras. Barcelona: Paidós.

Flecha, R., \& García Yeste, C. (2007). Prevención de conflictos en las comunidades de aprendizaje. Idea La Mancha: Revista de Educación de Castilla La Mancha, 4.

Freire, P. (1970). Pedagogía del oprimido. Madrid: Siglo XXI [V.O. Pedagogia do oprimido. Rio de Janeiro: Paz e Terra, 1970].

Gómez, J., Latorre, A., Sánchez, M., \& Flecha, R. (2006). Metodología Comunicativa Crítica. Barcelona: El Roure Ciencia.

Habermas, J. (1998). Facticidad y validez. Sobre el derecho y el Estado democrático de derecho en términos de teoría del discurso. Madrid: Morata (p.o. 1992).

Habermas, J. (1987). Teoría de la acción comunicativa. Volumen I: Racionalidad de la acción y racionalización social y Volumen II: 
Crítica de la razón funcionalista. Madrid: Taurus [V.O. Theorie des kommunikativen Handelns, 2 vols. Frankfurt am Main: Suhrkamp, 1981].

Hellison, D. (1995). Teaching Responsability Through Physical Activity. Champaign: Human Kinetics.

Hellison, D. (1999). Promoting Character Development through Sport. Rhetoric or Reality? New Designs for Youth Development, 15(1), 23-26.

Hellison, D. (2003). Teaching responsibility through physical activity(2 ed.). Champaign: Human Kinetic.

INCLUD-ED Conssortiu. (2009). Actions for success in schools in Europe. Bruselas: European Commission.

Janssen, M., Toussaint, H.M, Van Willem, M., \& Verhagen, E.A.L.M. (2011). PLAYgrounds: Effect of a PE playground program in primary schools on PA levels during recess in 6 to 12 year old children. Design of a prospective controlled trial. BMCPublic Health, 11. doi: $10.1186 / 1471-2458-11-282$

Jiménez, P.J., \& Durán, L. J. (2004). Propuesta de un programa para educar en valores a través de la actividad física y el deporte. Apunts, 77, 25-29.

Kain, J., Leyton, B., Cerda, R., Vio, F., \& Uauy, R. (2009). Two-year controlled effectiveness trial of a school-based intervention to prevent obesity in Chilean children. Public Health Nutrition, 12(9), 14511461. doi: $10.1017 / S 136898000800428 X$

Kirikiades, L., \& Valls, R. (2013). The power of Interactive Groups: how diversity of adults volunteering in classroom groups can promote inclusion and success for children of vulnerable minority ethnic populations. Cambridge Journal of Education, 43(1), 1733.

Koh, H.K., Piotrowski, J.J., Kumanyika, S., \& Fielding, J. (2011). Healthy People: A 2020 Vision for the Social Determinants Approach. Health Education \& Behavior, 38, 6, 551-557. doi: 10.1177/1090198111428646

Macazaga, A.M., Rekalde, I., \& Vizcarra, M.T. (2013). ¿Cómo encauzar la agresividad? Una propuesta de intervención a través de juegos y deportes. Revista Española de Pedagogía, 255, 263-276.

Mead, G.H. (1973). Espíritu, persona y sociedad: desde el punto de vista del conductismo social. Barcelona: Paidós [V.O. Mind, Self and Society. Londres: The University of Chicago Press, 1934].

Molina, F. (2005). Nuevos conflictos sociales y su presencia educativa. Análisis sociológico y reflexiones para la intervención. Cultura y Educación, 17 (3), 213-223.

Monjas, M.I. (2008). Como promover las convivencia. Programa de asertividad y habilidades sociales. (PAHS). Madrid: Cepe.

Ortí, J. (2003). La resolución de conflictos en la Educación Física. Tándem. Didáctica de la Educación Física, 13, 40-50.

Pappa, E., Kontodimopoulos, N., Papadopoulos, A.A., \& Niakas, D.
(2009). Assessing the socio-economic and demographic impact on health-related quality of life: evidence from Greece. International Journal of Public Health, 54(4), 241-249. doi: 10.1007/s00038009-8057-x

Telford, R. D., Bass, S. L., Budge, M. M., Byrne, D. G, Carlson, J. S., Coles, D., ... Waring, P. (2009). The lifestyle of our kids (LOOK) project: outline of methods. Journal of science and medicine in sport / Sports Medicine Australia, 12(1), 156-63. doi:10.1016/ j.jsams.2007.03.009

Tellado, I., \& Sava, S. (2010). The role of non-expert adult guidance in the dialogic construction of knowledge. Revista de psicodidáctica, 15(2), 163-176.

Tercyak, K.P., Abraham, A.A., Graham, A.L., Wilson, L.D., \& Walker, L.R. (2009). Association of Multiple Behavioral Risk Factors with Adolescents Willingness to Engage in eHealth Promotion. Journal of Pediatric Psychology, 34(5), 457-469. doi: 10.1093/jpepsy/jsn085

Thorburn, M., Carse, N., Jess, M., \& Atencio, M. (2011). Translating change into improved practice: Analysis of teachers' attempts to generate a new emerging pedagogy in Scotland. European Physical Education Review, 17(3), 313-324. doi: 10.1177/ 1356336X11416727

Vargas, J., \& Flecha, R. (2000). El aprendizaje «dialógico» como experto en resolución de conflictos. Contextos educativos(3), 81-88.

Vizcarra, M.T. (2004). Análisis de una experiencia de formación permanente en el deporte escolar a través de un programa de habilidades sociales, (serie tesis doctorales). Bilbao: UPV/EHU.

Velázquez Callado, C. (2003). El aprendizaje cooperativo en Educación Física: qué, para qué, porqué y cómo. In Ampliando horizontes a la cooperación: actas del III Congreso Estatal y I Iberoamericano de Actividades Físicas Cooperativas. Valladolid: La Peonza.

Velázquez Callado, C. (2004). Las actividades físicas cooperativas. Una propuesta para la formación en valores a través de la educación física en las escuelas de educación básica. México: Secretaría deEducación Pública.

Vizcarra, M.T., Macazaga, A., Perera, S., Maiztegui, A., Arostegui, B., \& Gasituaga, L. (2007). Un plan integrado para la convivencia apoyado en la resolución de conflictos. In J.J. Goicochea \& M.T. Vizcarra (Eds.), Los retos actuales en la investigación educativay la formación profesionalizadora de los estudios de magisterio (pp. 356-364). Vitoria-Gasteiz: Idazkide.

Vizcarra, M.T., Macazaga, A.M., \& Rekalde, I. (2013). El proceso de acompañamiento en la construcción participativa de una normativa. Revista de Orientación Psicopedagógica, 24 (1), 110-120.

Vygotsky, L.S. (1996). El desarrollo de los procesos psicológicos superiores. Barcelona: Crítica [V.O. Mind in Society: The development of higher psychological processes. Cambridge, MA: Harvard University Press, 1978].
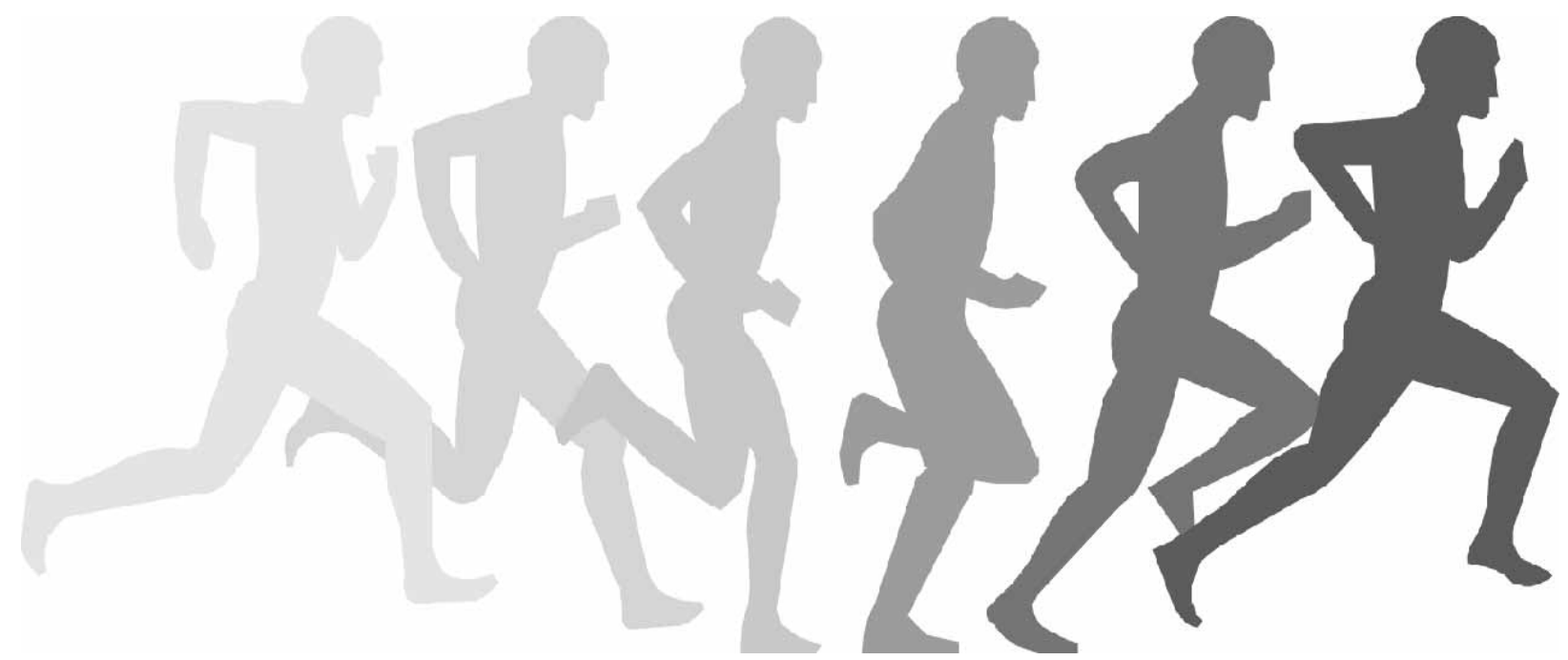